\title{
Bambara et français-tirailleur
}

Une analyse de la politique linguistique au sein de l'armée coloniale française : la Grande Guerre et après

\section{Cécile Van den Avenne}

\section{(2) OpenEdition \\ 12 Journals}

\section{Édition électronique}

URL : https://journals.openedition.org/dhfles/1115

DOI : 10.4000/dhfles. 1115

ISSN : 2221-4038

Éditeur

Société Internationale pour l'Histoire du Français Langue Étrangère ou Seconde

\section{Édition imprimée}

Date de publication : 1 décembre 2005

Pagination : 123-150

ISSN : 0992-7654

\section{Référence électronique}

Cécile Van den Avenne, "Bambara et français-tirailleur », Documents pour l'histoire du français langue étrangère ou seconde [En ligne], 35 | 2005, mis en ligne le 03 novembre 2014, consulté le 27 mai 2021. URL : http://journals.openedition.org/dhfles/1115 ; DOI : https://doi.org/10.4000/dhfles.1115

Ce document a été généré automatiquement le 27 mai 2021.

(c) SIHFLES 


\section{Bambara et français-tirailleur}

Une analyse de la politique linguistique au sein de l'armée coloniale

française : la Grande Guerre et après

\section{Cécile Van den Avenne}

1 C'est en 1857 qu'est créé le premier régiment de tirailleurs sénégalais; en 1914, à la veille de la Première Guerre mondiale, l'effectif maintenu en Afrique occidentale française (AOF) représente environ 12000 hommes. Peu avant la guerre, le général Mangin, qui publie en 1910 son ouvrage célèbre La Force noire (Mangin 1910), développe un projet d'armée noire propre à s'engager dans les conflits extérieurs et on décide la création d'une "réserve indigène » dans chaque colonie prête à répondre à l'urgence. S'opère alors une mutation dans le recrutement et dans la nature des unités noires de l'armée coloniale: de troupes de soldats de métier qu'elles étaient, elles deviennent troupes de simples conscrits, réquisitionnés souvent sous la contrainte, phénomène qui s'accentue avec l'engagement des bataillons de tirailleurs sénégalais dans la Grande Guerre. Ainsi, s'ajoutant aux 31000 tirailleurs déjà mobilisés, on leva, en cinq campagnes de recrutement, 167000 hommes, la moitié venant du Haut-Sénégal et Niger, un cinquième de Guinée, $14 \%$ de Côte d'Ivoire, $13 \%$ du Sénégal, $7 \%$ du Dahomey ${ }^{1}$.

2 Les tirailleurs dits "sénégalais " sont donc en fait minoritairement sénégalais. Les Sénégalais originaires des Quatre Communes ${ }^{2}$ sont par ailleurs engagés dans les régiments de métropolitains en qualité de "citoyens" français. La diversité des origines des tirailleurs a pour conséquence une grande diversité linguistique au sein des bataillons. Sans compter le fait que 7 à $10 \%$ des effectifs sont composés de Blancs : les officiers, une partie des sous-officiers et les titulaires de spécialités techniques sont français. L'africanisation des cadres de l'armée ne dépasse guère le niveau des sousofficiers, et en 1916, les autorités militaires et civiles écartent encore toute idée de promotion d'indigènes aux grades d'officiers. La question de la communication est donc à la fois un problème horizontal et vertical : elle concerne à la fois la communication quotidienne entre tirailleurs et la question, plus directement cruciale, de la compréhension des ordres. Les différents documents que nous avons à notre disposition rendent compte de deux types de pratique permettant 
l'intercommunication: d'une part l'usage de parlers véhiculaires, d'autre part l'usage de la traduction. Par ailleurs, ils révèlent, concernant le choix d'un véhiculaire, d'une hésitation et d'une pluralité de pratiques, une langue indigène pouvant être promue au rang de véhiculaire au sein de l'armée (c'est ce que nous verrons à propos du bambara), ou le français (dans une version éventuellement simplifiée) pouvant remplir ce rôle. Nous rendrons compte ici à la fois d'articulations et de tensions entre ces diverses pratiques qui se révèlent à la lumière de l'engagement dans la Grande Guerre, et nous tâcherons d'expliciter les conceptions anthropologiques qui les sous-tendent, dans la mesure où l'on peut considérer les pratiques militaires comme largement influencées par la manière dont le savoir ethnographique est en train de se constituer, au début du $\mathrm{XX}^{\mathrm{e}}$ siècle, en et sur l'Afrique de l'Ouest.

Illustration 1 : extrait d'une carte légendée « Carte des races de l'Afrique Occidentale Française fournissant des tirailleurs sénégalais » et publiée dans La dépêche coloniale illustrée, février 1917 : "L'Afrique occidentale française et les troupes noires".

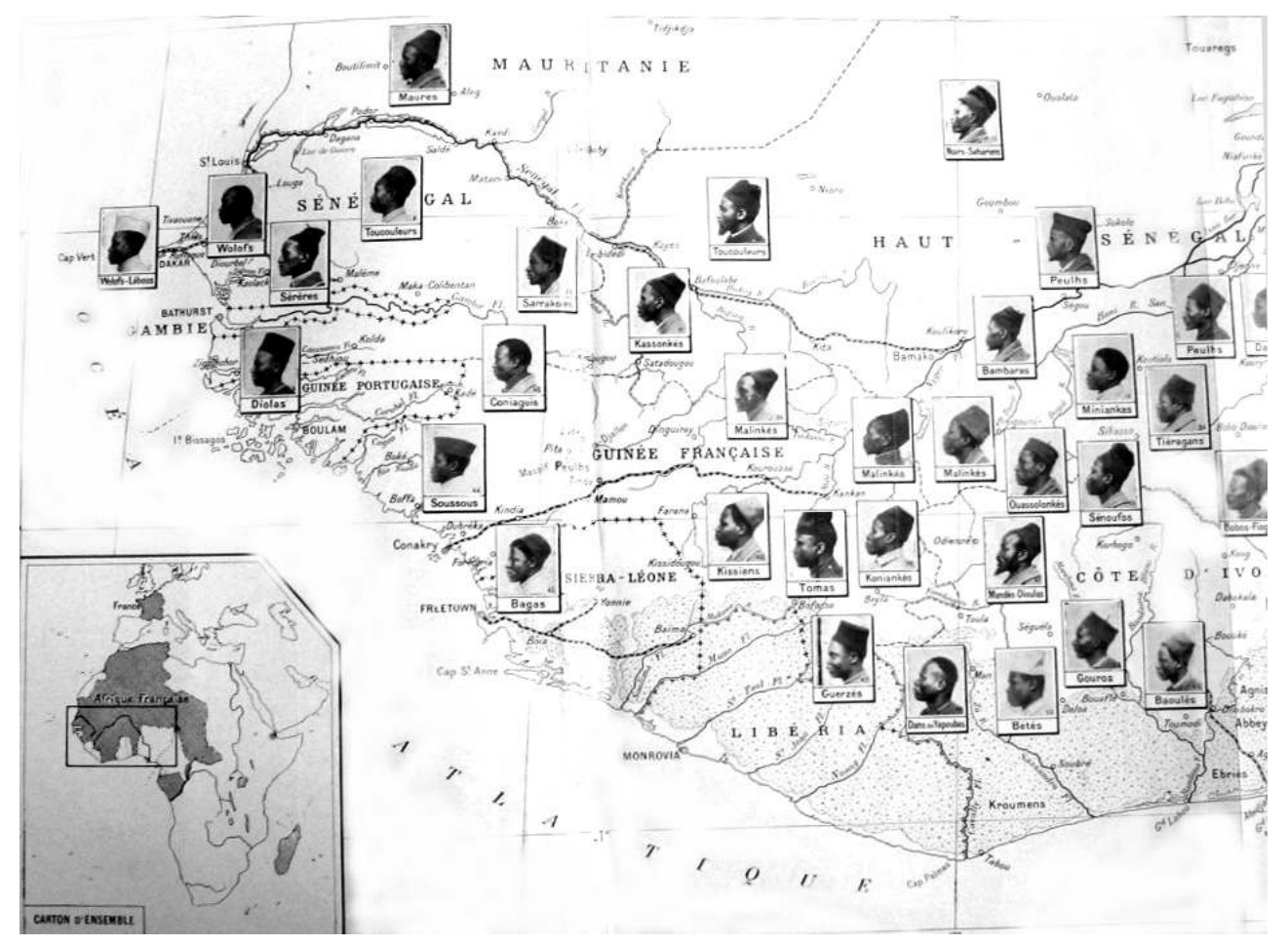

\section{Quelle langue véhiculaire pour les tirailleurs? Bambara et/ou petit-nègre}

Si la diversité linguistique est grande au sein des bataillons de tirailleurs sénégalais, une langue cependant domine et a acquis un certain rôle véhiculaire : le bambara, ou bamanankan, langue du groupe mandé parlée dans une zone qui va du Haut-Sénégal au Soudan (Mali actuel) en passant par la Guinée. L'armée ne fait que récupérer et accentuer les usages véhiculaires de cette langue, langue de commerce avant la conquête coloniale et, de ce fait, langue des interprètes utilisés pendant la conquête coloniale. Il se trouve par ailleurs que le bambara est une des langues d'Afrique de l'Ouest les plus anciennement décrites ${ }^{3}$. 
Dans son Manuel à l'usage des troupes opérant au Soudan français et plus particulièrement en zone saharienne (Bulletin 1922 : 590-648), le Colonel Mangeot précise la manière dont certaines autorités militaires envisagent la langue bambara comme langue véhiculaire au sein de l'armée :

il serait bon que chacun possède des notions suffisantes de langue bambara. De plus en plus, celle-ci s'affirme comme langue communément parlée par tous les tirailleurs ; qu'ils viennent de la Côte d'Ivoire, du Dahomey, du Mossi, de la Guinée ou du Sénégal, nos tirailleurs appelés ou engagés apprennent peu à peu les rudiments du bambara et rares sont ceux qui quittent le service sans le parler couramment.

Nos cadres européens doivent suivre cet exemple. La langue est facile, le vocabulaire est sobre, la grammaire simple. (ibid. : 611)

On peut émettre l'hypothèse que, mis à part pour les quelques bambarophones de langue première ou seconde, qui devaient exister dans les régiments, le bambara utilisé était un bambara rudimentaire et simplifié, tout comme l'était la variété de français utilisée, dont nous allons rendre compte par la suite. Les manuels à disposition des Occidentaux sont avant tout des glossaires et ne rendent compte (et encore de manière extrêmement simplifiée) que de la construction de quelques types de phrases simples. Ils proposent par ailleurs des énoncés types, sur le modèle des guides de conversation, tels ceux publiés à l'usage des touristes jusqu'à présent. C'est notamment le cas du manuel rédigé en 1910 par Moussa Travélé, locuteur de langue bambara et interprète titulaire de $2^{\mathrm{e}}$ classe de la colonie Haut-Sénégal Niger, dont Maurice Delafosse, dont nous reparlerons, alors ancien administrateur du cercle de Bamako et également auteur d'un manuel de bambara (Delafosse 1901), écrit, non sans condescendance, dans la préface à l'ouvrage, que l'auteur a « cherché simplement à permettre aux Européens résidant en pays bambara d'arriver facilement et vite à bien parler et à se faire bien comprendre » (Travélé 1910). Ce manuel propose notamment une conversation type intitulée " Tournée de recensement ", qui permet notamment de dire : "Où est le chef du village? As-tu fini de payer ton impôt ? Vous regarderez dans les cases s'il n'y a personne de caché », soulignant que ce manuel est avant tout à destination des administrateurs coloniaux.

6 Un autre manuel, beaucoup plus précis, est un ouvrage militaire rédigé spécifiquement à l'usage des troupes coloniales (Ferrage 1918) par un Père Blanc, le Père P.-M. Ferrage, missionnaire au Soudan et interprète au $74 \mathrm{e}$ bataillon de tirailleurs sénégalais. L'auteur écrit dans la préface à son ouvrage :

Bien que la plupart de nos Tirailleurs Sénégalais sachent les mots les plus usuels de la langue française, il est quelquefois fort difficile, sinon impossible, de se faire comprendre d'eux autrement qu'en leur langue. C'est pourquoi, sur le désir exprimé par Monsieur le Colonel Julien, commandant le Groupe des Bataillons Sénégalais d'Algérie et encouragé par sa haute bienveillance, nous publions ce «Petit Manuel français-bambara ».

Notre but, en le présentant aux nombreux gradés européens qui servent dans les troupes noires, n'est pas de leur apprendre une langue avec laquelle une longue pratique pourrait seulement les familiariser, mais bien de leur donner un instrument facile [...].

7 Ce manuel décrit précisément le fonctionnement des différents énoncés simples en bambara, mais ne rend pas compte de la construction de la phrase complexe. Il propose par ailleurs différents dialogues en bambara, qui vont des salutations, souhaits, et 
remerciements à des dialogues en contexte militaire (à la caserne, en colonne, des tranchées...).

Illustration 2 : couverture de : P.-M. Ferrage (1918) Petit manuel français-bambara à l'usage des troupes noires, Paris : Imprimerie-Librairie militaire L. Fournier.

P. M. FERRMG

\section{Petit Manuel}

Français-Bambara

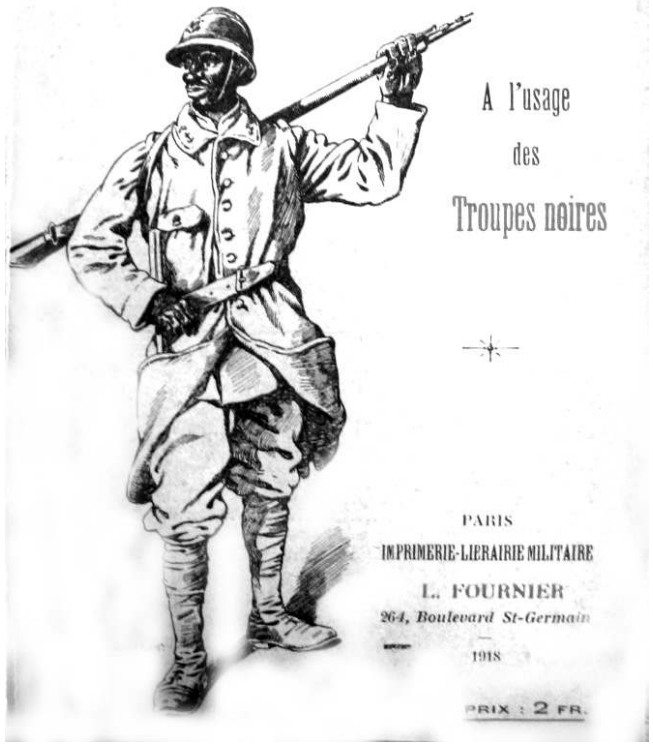


Illustration 3 : extrait de : P.-M. Ferrage (1918), Petit manuel français-bambara à l'usage des troupes noires, Paris : Imprimerie-Librairie militaire L. Fournier.

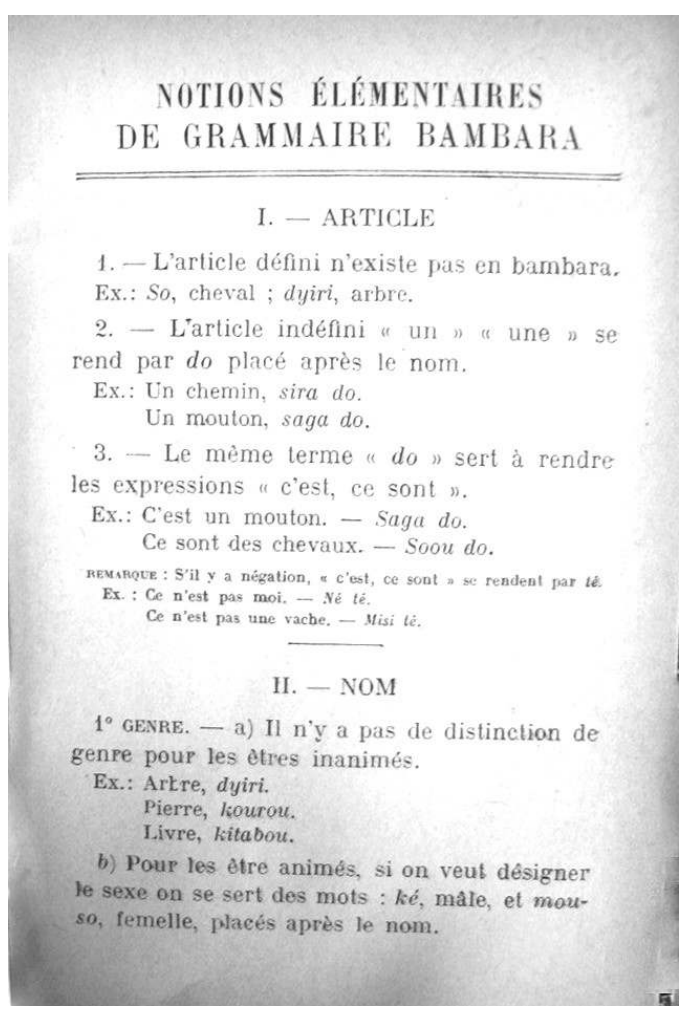

On sait finalement peu de choses sur la pratique du bambara dans les régiments de tirailleurs et sur la compétence et des tirailleurs non natifs et des officiers français dans cette langue. Des sources littéraires rendent compte d'une connaissance rudimentaire de cette langue ; il s'agit des différents romans qui retracent les aventures de tirailleurs dans les tranchées de Verdun. En effet, à partir de la fin de la guerre de 1914-1918 et ceci jusqu'à la Seconde Guerre mondiale, sont publiés plusieurs dizaines de romans mettant en scène des tirailleurs sénégalais. Le succès éditorial de ce genre de romans s'appuie sur la popularisation de ces tirailleurs sénégalais, dont les exploits guerriers sont magnifiés par la presse durant la Première Guerre mondiale, et qui nouent des liens avec la population civile lors de leurs hivernages méditerranéens, à Marseille, Fréjus, Nice et Menton. Si certains de ces romans peuvent être dits de "seconde main », mais parfois documentés auprès d'acteurs sociaux au fait des réalités coloniales (administrateurs ou militaires), nombreux sont ceux publiés par d'anciens officiers des régiments coloniaux. On repère dans ces romans quelques mises en scène de microinteractions en bambara.

9 On peut en lire un exemple dans l'ouvrage d'un mémorialiste (Séché 1919), mise en forme d'une compilation de divers documents qui rendent compte de différents épisodes de l'engagement des tirailleurs sénégalais dans la Grande Guerre. Dans un chapitre intitulé « Les Sénégalais à Berry-au-Bac » qui retrace l'attaque de la cote 91 le 15 octobre 1914, et dont le récit est donné comme la transcription d'un récit oral que lui a fait Albert Mallet, capitaine d'une compagnie de tirailleurs ${ }^{4}$, Alphonse Séché insère de courts dialogues où apparaissent quelques énoncés en bambara :

- Yeut'nant, yeut'nant, implore une voix, ki kana (viens ici)

- Djondo? (Qui est-ce ?)

- Sibiri 
Sibiri, un magnifique Bambara et l'un des meilleurs tireurs. (ibid. : 89)

et un peu plus loin :

Moussa interroge un tirailleur

- a sara ! - il est mort. (ibid. : 91)

10 Mais ces romans mettent davantage complaisamment en scène des interactions en « français-tirailleur », dit aussi « petit nègre ».

\section{La fabrication du français-tirailleur}

11 On désigne par le terme de «français-tirailleur » la forme de français parlée par les tirailleurs dits sénégalais au sein de l'armée coloniale française. Robert Chaudenson en donne cette définition : il s'agit d'un

pidgin quelque peu artificiel, qui procède à la fois de variétés approximatives produites par des locuteurs en situation exolingue et de généralisations didactiques réputées faciliter et accélérer l'apprentissage de ce français minimal. (2003: 54)

Le « français-tirailleur » semble donc être le produit d'un double processus : d'une part un processus d'approximation et de simplification co-produit en interaction par les officiers et leurs soldats indigènes, processus commun à la fois aux pidgins, soit ces types de parlers aux fonctionnalités réduites (commerce, relations ancillaires) et à ce que l'on désigne par le terme de foreigner talk, soit cette variété simplifiée produite par des natifs à l'intention d'un interlocuteur étranger à sa langue; d'autre part, un processus de stabilisation à travers un enseignement plus ou moins formalisé, comme le laisse supposer la publication en 1916 d'un manuel intitulé Le français tel que le parlent nos tirailleurs sénégalais (Anonyme 1916), ouvrage qui se donne tout à la fois comme description d'un parler créé par les tirailleurs au contact de leurs instructeurs européens et comme manuel permettant aux gradés européens d'apprendre ce parler afin de «se faire comprendre en peu de temps, de leurs hommes, de donner à leurs théories une forme intelligible pour tous et d'intensifier ainsi la marche de l'instruction » (ibid. : 5).

Le/les auteurs de ce manuel sont anonymes. Pour tâcher de comprendre dans quel contexte il a pu être écrit, un certain nombre de points doivent être éclaircis, qui ont trait à la nature du savoir linguistique qui se constitue au début du $\mathrm{XX}^{\mathrm{e}}$ siècle en et sur l'Afrique occidentale française (conjointement car la constitution du savoir linguistique a été le fait avant tout des administrateurs coloniaux). Le cas le plus célèbre est celui de Faidherbe, gouverneur du Sénégal entre 1854 et 1865, à qui l'on doit, entre autre, les premières descriptions des langues peul, soninké, wolof et sérère, publiées entre 1859 et 1887 .

14 Ce fascicule témoigne d'une connaissance, même approximative, du bambara, dont nous avons dit qu'il a pu constituer une forme de véhiculaire au sein de l'armée. Il est ainsi précisé dans les préliminaires de l'ouvrage, la règle de base pour produire du français-tirailleur : «Donner toujours à la phrase française la forme très simple qu'a la phrase dans tous les dialectes primitifs de notre Afrique Occidentale » (ibid.: 5), et les différents exemples donnés de " dialectes primitifs » sont tous des exemples bambara. Le recours à des exemples en bambara sert donc à cautionner la fabrication de ce parler. Un exemple, au chapitre « Article » :

Dans les dialectes de notre A.O.F., l'article n'existe pas : pour désigner un objet, on en indique seulement le nom. Exemple : falo signifiera en bambara, l'âne, un âne, âne. 
Il n'y aura donc pas lieu dans les phrases d'employer d'article.

de l'enseignement du «français-tirailleur », sans mentionner l'influence de Maurice Delafosse, administrateur colonial et linguiste (1870-1926). Maurice Delafosse, en effet, est considéré comme étant le premier à avoir donné une description linguistique du " petit-nègre ", " simplification naturelle et rationnelle de notre langue si compliquée " (Delafosse $1904: 263$ ) et à avoir promu ce parler au rang de «langue »5. Partant d'une représentation des populations noires comme primitives, et des langues d'Afrique subsaharienne comme « langues simples » il écrit :

Comment voudrait-on qu'un Noir, dont la langue est d'une simplicité rudimentaire et d'une logique presque toujours absolue, s'assimile rapidement un idiome aussi raffiné et illogique que le nôtre ? C'est bel et bien le Noir - ou, d'une manière plus générale, le primitif - qui a forgé le petit-nègre, en adaptant le français à son état d'esprit. (Delafosse $1904: 264$ )

Et il finit son introduction sur ces mots :

si nous voulons nous faire comprendre vite et bien, il nous faut parler aux Noirs en nous mettant à leur portée, c'est-à-dire leur parler petit-nègre.

La description syntaxique que fait Delafosse, dans son ouvrage, du petit-nègre tient en 20 lignes :

18 - emploi des verbes à leur forme la plus simple (infinitif de la première conjugaison, participe passé, impératif)

19 - négation exprimée uniquement par le forclusif « pas »

20 - suppression des distinctions de genre et de nombre

21 - suppression de l'article ou agglutination de l'article au substantif

22 - usage considérable du verbe "gagner " et des locutions "y a ", " y en a » comme semi-auxiliaires

23 - emploi de l'adverbe « là » comme démonstratif

24 - suppression des prépositions «à » et «de » et remplacement fréquent par la préposition " pour ».

25 S'y ajoutent des remarques phonétiques: e muet final remplacé par une voyelle prononcée (caissou pour caisse), phénomènes d'harmonisation vocalique (piti pour petit), remplacement des constrictives dorso-vélaires par des constrictives dentales (semise pour chemise, zenou pour genou).

26 Il est très probable que la première description de Delafosse a servi de base aux auteurs du manuel militaire (on pourrait tout aussi bien supposer qu'il est l'auteur lui-même du fascicule, lui qui était polygraphe $\left.{ }^{6}\right)$. On peut noter également que Delafosse est l'auteur d'un des premiers manuels (sinon le premier) de langue mandé (famille des langues mandé ou mandingue, dont fait partie le bambara).

27 Nous ne reviendrons pas sur la description syntaxique de ce parler proposé par le manuel (elle a été faite par d'autres et nous renvoyons ici aux analyses de Manessy et Houis); elle développe en fait la description rudimentaire de Delafosse en l'exemplifiant par des énoncés propres à être prononcés dans le cadre d'une instruction militaire (du type : «Patrouille y a parti vite», ibid. : 31). Sont ainsi proposés des tableaux de traduction français standard/français tirailleur. 
Illustration 4 : extrait de : Le français tel que le parlent nos tirailleurs sénégalais, anonyme, Imprimerie Militaire Universelle L. Fournier, Paris : 1916.

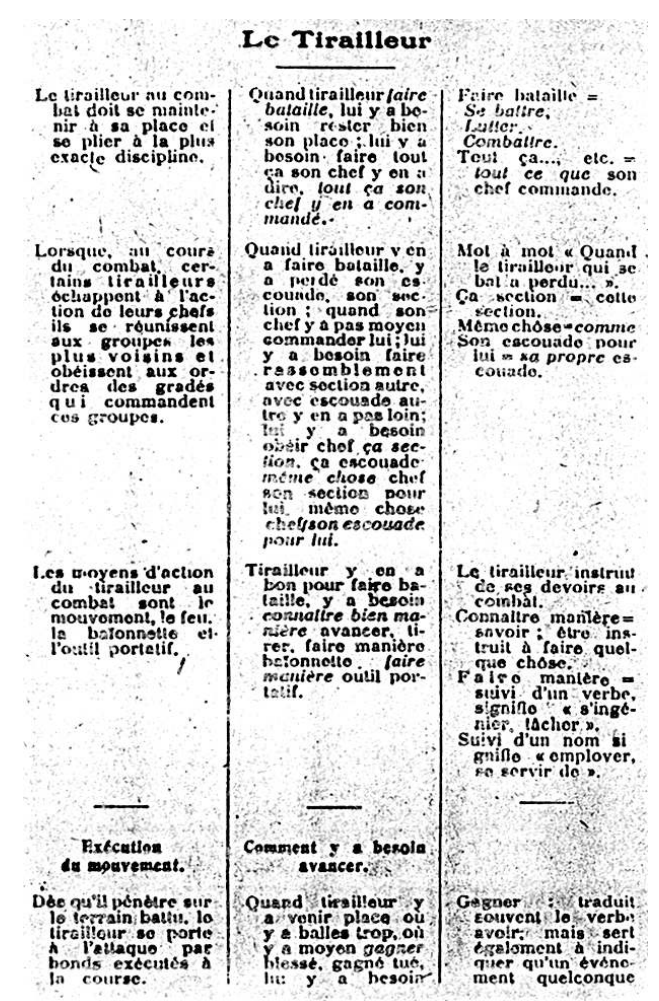

28 Deux autres éléments peuvent être pris en considération pour rendre compte de l'apparition de cet usage du "français simplifié » (c'est ainsi qu'est nommée cette variété dans les notes du ministère de la Guerre, voir ci-dessous). L'un concerne le choix de la variété linguistique, l'autre les méthodes d'acquisition envisagées.

En ce qui concerne le choix de la variété linguistique, et le fait qu'elle peut être décrite comme une langue auxiliaire artificielle, produit d'une simplification et d'une systématisation, on peut noter qu'il paraît s'inscrire ainsi dans ce mouvement qui, à partir de la fin du XIX ${ }^{\mathrm{e}}$, voit la création totalement artificielle de diverses langues universelles, qui vont, comme le volapük créé en 1879 ou l'espéranto créé en 1887, devenir d'effectives langues de communication. Dans ce contexte, le français-tirailleur peut faire figure d'espéranto à usage colonial (à la réserve près que ces diverses langues artificielles n'étaient pas pensées quant à elles pour les relations avec des «indigènes non civilisés ", mais pour, ainsi que le spécifie la Délégation pour l'adoption d'une langue auxiliaire internationale constituée en 1901, « servir aux relations habituelles de la vie sociale, aux échanges commerciaux et aux rapports scientifiques et philosophiques » (cité par Auroux 2000 : 380).

Quant aux méthodes d'acquisition envisagées, elles font écho à l'apparition et au développement de méthodes d'apprentissage des langues étrangères, par la méthode directe, notamment la célèbre méthode Berlitz (explicitement citée dans une note du ministère de la Guerre, voir ci-dessous). La méthode directe est celle prescrite dans les programmes et instructions du ministre de l'Instruction publique pour l'enseignement des langues vivantes dans les établissements secondaires, elle consiste en une méthode qui n'utilise pas la langue maternelle de l'élève, c'est-à-dire qui ne passe pas par la traduction, mais par une mise en relation directe entre référent et signifié. Un extrait 
du Manuel à l'usage des troupes opérant au Soudan français et plus particulièrement en zone saharienne, du Colonel Mangeot (Bulletin 1922: 613), permet de rendre compte de ce que l'on entend par méthode directe au sein de l'armée :

Dans tous nos régiments de tirailleurs sénégalais, il a toujours été de tradition de consacrer quelques heures par semaine, à l'enseignement de notre langue. La méthode est simple. On commence par leur apprendre en français la nomenclature des vêtements, des armes qui leur sont confiés ; à l'exercice, on enseigne le sens des commandements, les noms des objets rencontrés à la surface du sol. Peu à peu on assemble les mots en phrases simples, courtes.

Quand il connaît ces rudiments, les progrès du tirailleur sont rapides.

Le soir au camp, les anciens, ceux qui parlent couramment notre langue, font la leçon.

Ici encore, c'est le sentiment de l'orgueil qui domine, l'ancien veut étonner la recrue par ses connaissances et la recrue a le désir de parler «même chose l'officier français ». Il faut cultiver cet orgueil.

31 Ce qui mis en récit de façon volontairement caricaturale donne ceci, où l'on voit à l'œuvre un mélange de méthode directe et de pratique de traduction, en l'occurrence en bambara :

Puis vient la leçon de français. Les hommes sont répartis par petits groupes à la tête desquels il n'est pas toujours possible de mettre un moniteur européen ou un indigène qualifié de la valeur de Samba. Aussi faut-il faire attention à nos instructeurs bénévoles qui commettent des erreurs amusantes. Voyez le groupe du caporal Baba. Les tirailleurs sont assis en cercle, le regard attentif. Baba au milieu, debout, indique la leçon du jour : « Les alimaux comestiques. » Il annonce ce titre, puis s'adresse aux tirailleurs: «Afo anofé » (répétez). De vagues sons sont émis, Baba continue :

- La premier ça y en a la bef (le bœuf)

- Afo

Les tirailleurs répètent « la bef » sans comprendre.

Baba explique :

- la bef a bekakan « missi »

Les tirailleurs ont compris que le « missi et « la bef » sont le même animal.

Baba continue :

- La deuxième y en a « la miton » (le mouton).

Même interrogation. Les tirailleurs répètent « la miton » et Baba explique que «la miton » c'est le «saga ».

Alphonse Séché, mémorialiste dont nous avons parlé ci-dessus, visitant le camp d'hivernage de Fréjus, fait ce récit de la façon d'enseigner des rudiments de français, rendant compte et de l'indigence pédagogique (usage de la répétition), et des difficultés linguistiques :

Ailleurs un caporal explique : «Dans une semaine il y a sept jours, qui sont : lundi, mardi, marcredi, - il appuie fortement sur l'a, - jeudi, venderdi, samedi, dimanche. » [...] Le caporal fait répéter les mêmes mots indéfiniment. Sa patience n'a d'égale que la difficulté des Sénégalais à retenir ce qu'on leur enseigne. Quelquefois l'instructeur parle en sa langue. Les tirailleurs n'étant pas tous de même race, il arrive qu'ils ne comprennent pas davantage le bambara, le toucouleur ou le soussou, que le français. Je plains les sous-officiers européens, écrit Séché, réduits, pour la plupart, à s'exprimer en petit nègre, même lorsqu'ils donnent des instructions aux gradés indigènes. (Séché, 1919 : 72-73) ${ }^{8}$ 


\section{Interprètes en langues indigènes ou usage du français simplifié : problématiques linguistiques pendant la guerre de 1914-1918}

L'engagement des bataillons de tirailleurs sénégalais dans la guerre de 1914-1918 révèle des problématiques linguistiques assez similaires à celles qui émergent de la prise en compte des dialectophones au sein des régiments français9. En effet si les "vieux" tirailleurs ont eu le temps d'acquérir des rudiments ou plus de la langue française, les nouvelles recrues, quant à elles, peuvent ne pas parler français du tout.

Si différents documents attestent de pratiques de traduction sur le front, les conséquences linguistiques de la guerre seront l'intensification de l'apprentissage du français. Les autorités militaires se prononcent finalement, on le verra, contre la création d'un corps d'interprètes et pour l'intensification de l'apprentissage du français. Les pratiques de traduction ne sont cependant pas absentes, les camps et les unités étant dotés de traducteurs blancs (administrateurs coloniaux, mais aussi Pères Blancs) et noirs (des sous-officiers).

Ainsi, le journal de marche des opérations du $61^{\mathrm{e}}$ bataillon de tirailleurs sénégalais fait mention du rôle d'un traducteur avant un combat dans la Somme, dans un rapport des 9 et 10 juillet 1916, en ces termes :

Le chef de bataillon fait traduire aux tirailleurs par l'intermédiaire des adjudants indigènes quelques paroles dans le but de leur fixer nettement l'objectif et de stimuler leur $\operatorname{ardeur}^{10}$.

La langue utilisée n'est pas spécifiée. On peut supposer qu'il s'agissait du bambara ; la mention d'une traduction faite par plusieurs adjudants indigènes peut laisser supposer également que la traduction s'est faite en plusieurs langues, peut-être le peul, le wolof et le mossi, groupes représentés dans ce bataillon (d'après l'examen des patronymes), et langues servant diversement de véhiculaires pour groupes originaires de mêmes zones géographiques mais non de mêmes groupes linguistiques.

Par ailleurs, dans deux notes rédigées pour le ministre de la Guerre ${ }^{11}$, le général Famin, général directeur des troupes coloniales, indique d'une part que plusieurs soldats provenant d'AOF (il s'agit de soldats européens) et parlant couramment le bambara ont été placés dans les bataillons sénégalais en qualité d'infirmiers interprètes, et que d'autre part les infirmiers européens auront le grade de caporal ou sergent « sous la réserve qu'ils parlent couramment la langue indigène et puissent, le cas échéant, servir d'interprètes ". 
Illustration 5 : dessin extrait de : La dépêche coloniale illustrée, février 1917 : « L'Afrique occidentale française et les troupes noires ".

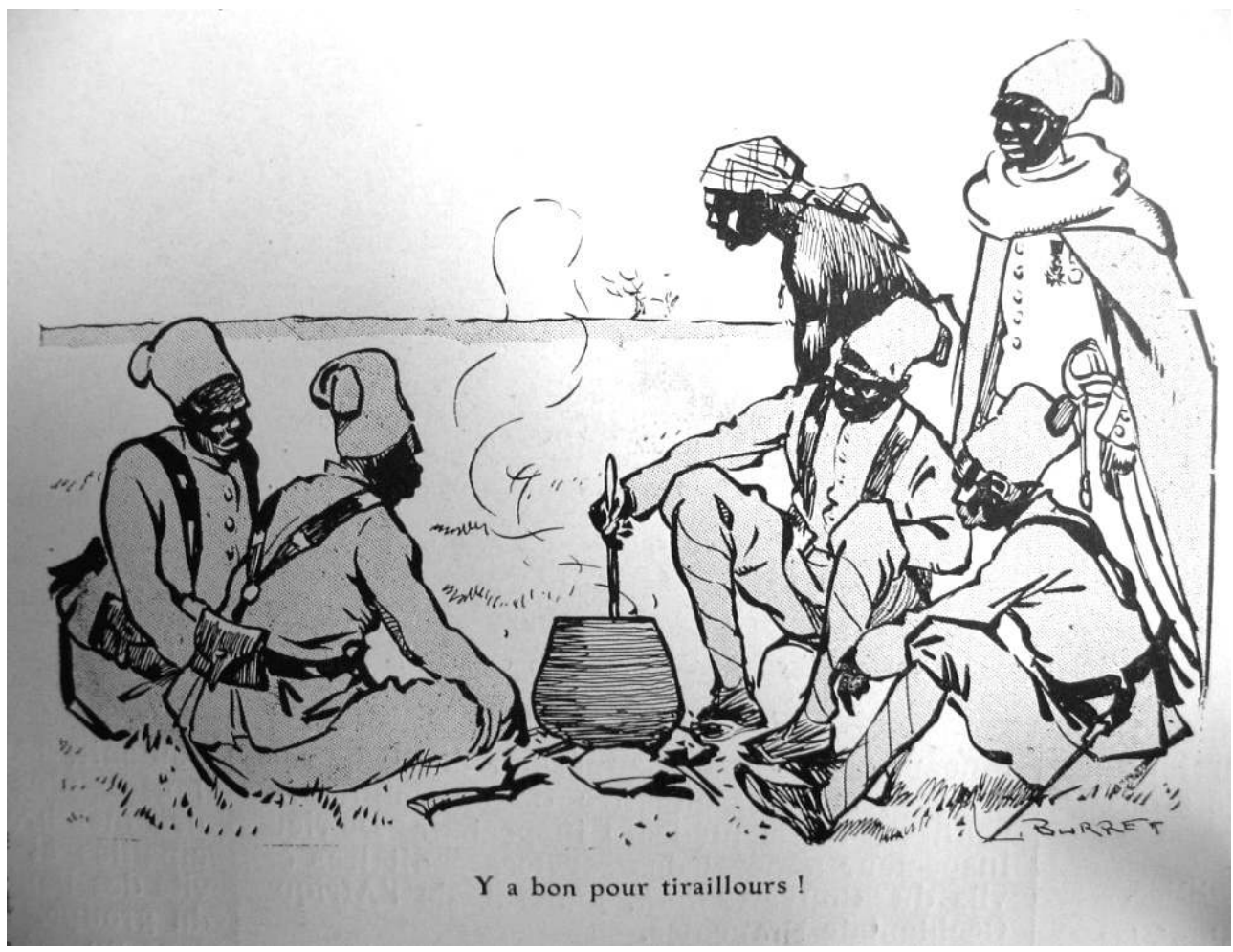

La prise de conscience de la pluralité linguistique au sein des bataillons de tirailleurs sénégalais, et de la maîtrise imparfaite de la langue française par les tirailleurs, a donné lieu à un débat au sein même du cabinet Clemenceau, débat dont nous allons retracer la chronologie.

Le 8 octobre 1917, le président du Conseil, ministre de la Guerre, fait suivre au général inspecteur des contingents indigènes, une note signée du général Mas, directeur des troupes coloniales, qui signale que :

Les bataillons sénégalais comprennent des tirailleurs, de races diverses, ne parlant pas la même langue. Il m'a été signalé que la dispersion, entre les unités, de tirailleurs ne parvenant pas à se faire comprendre des gradés, ni à les comprendre, présentait parfois des inconvénients

et préconisant qu'

Il y aurait peut-être avantage à grouper en escouades ou en demi-sections sous les ordres de gradés de même race, si possible, les tirailleurs ainsi isolés.

La réponse du général Pineau, inspecteur des contingents indigènes des troupes coloniales, souligne l'inconvénient d'une telle mesure : elle serait d'une part difficile à appliquer pour «certaines races peu connues et peu nombreuses » et d'autre part, elle aurait comme résultat négatif la " possibilité d'entente d'hommes du même groupe » et une "plus grande facilité d'adresser des réclamations collectives ${ }^{12}$. Le général Pineau préconise de :

former le plus possible de gradés de diverses races, en tenant compte de l'effectif de chacune de ces races et surtout pour enseigner les éléments de notre langue, indispensable pour permettre d'exercer le commandement des indigènes sans avoir recours à une interprétation toujours plus ou moins défectueuse ajoutant : 
J'ai relevé plus de cinquante dialectes divers et dans ces conditions je ne vois qu'un seul moyen de commandement possible : imposer une langue unique, la nôtre. création d'un corps spécial d'interprètes, arguant du très grand nombre de langues parlées en AOF (plus de soixante), du fait que seuls quelques rares Européens parlent deux ou trois d'entre elles, et qu'en outre les langues les plus répandues (à savoir le bambara, le peul, le mossi et le wolof) sont, selon lui, celles qui comptent le plus d'indigènes parlant français. De ce fait: "la diversité des idiomes parlés par les tirailleurs oblige à leur donner une langue commune, et cette langue ne peut être que le français ». le même sens : opposition à la création d'un corps d'interprètes et utilisation dudit «français simplifié ». Par exemple, le général Mazillier, commandant le $1^{\text {er }}$ corps d'Armée coloniale, écrit au ministre de la Guerre, une lettre datée du 27 avril 1918, dans laquelle il note :

dans les compagnies, les indigènes ne se comprenant pas entre eux, s'efforcent de connaître rapidement quelques éléments de français, qui leur sert de langue commune

et il ajoute :

Les dialectes soudanais, à deux ou trois exceptions près, n'ont qu'un nombre de mots très limités qui suffisent aux indigènes. Ceux-ci apprennent très rapidement les mots français correspondants et les verbes et composent d'eux-mêmes le langage « petit-nègre » dont ils se servent avec nous et entre eux.

Le 17 juin 1918, dans le rapport qui fait suite à sa mission auprès de bataillons de tirailleurs sénégalais sur le front, monseigneur Lemaître rapporte qu'il a prononcé un 
discours qui a été traduit en bambara par son adjoint, ancien missionnaire au Soudan, et que ce discours a ainsi été très bien reçu. La mission Lemaître, accusée de répandre des erreurs sur les troupes de tirailleurs, est suspendue par Clemenceau en novembre 1918.

Ce qui se joue ici, et qui sous-tend cette question de politique linguistique, est en fait une querelle de l'Église et de l'État ${ }^{14}$.

En effet, au delà des différents arguments pratiques (pluralité des langues, difficulté à trouver des traducteurs compétents...), un argument fort joue en défaveur des langues indigènes: celui de l'accroissement possible de l'autorité des Pères Blancs. Pour le général Mangin, la volonté, émanant d'un religieux, de création d'un corps d'interprètes est le signe d'une volonté d'accroître, aux yeux des indigènes, le prestige des Pères Blancs, seuls Européens véritablement connaisseurs des langues indigènes et donc seuls qualifiés à servir d'interprètes. Le général Mangin souligne le refus constant des Pères Blancs d'endosser la tenue militaire lorsqu'ils servent d'interprètes et va jusqu'à les qualifier d'« agents étrangers nuisibles ».

\section{Le refus du « petit-nègre »}

C'est donc l'usage du français qui est finalement préconisé au sein de l'armée coloniale, français simplifié tout d'abord, mais qui va progressivement faire place au français standard. Très tôt en effet, des voix s'élèvent contre la pratique de ce jargon caricatural qu'est le petit-nègre ${ }^{15}$, et les différentes critiques émises vont aboutir à la décision au sein de l'armée d'abandonner cette pratique.

Un facteur important va aller dans le sens de la «francophonisation » des tirailleurs. A partir de 1915-1916 sont organisés des "camps d'hivernage » pour les tirailleurs sénégalais dans des casernes de la Côte d'Azur, ou sous tentes sur la plage au nord de Fréjus. Les effectifs qui séjournent dans ces camps sont croissants : 28000 hommes en mai 1916, 40000 en décembre, 45000 en 1917 (voir Michel 2003). Ces camps d'hivernage contribuent au côtoiement des tirailleurs et des populations civiles. Par ailleurs ils sont l'occasion d'une mise en place de différents apprentissages et notamment de cours de langue française ainsi que de cours d'alphabétisation en français. Le Comité d'Assistance aux Troupes Noires constitué le 24 mars 1915, dont le président est J. M. Le Cesne, vice-président de la Compagnie française de l'Afrique occidentale et qui compte en son sein de nombreuses personnalités coloniales ayant des intérêts économiques en AOF, préconise l'enseignement des "premiers rudiments de l'enseignement scolaire » aux tirailleurs (La dépêche coloniale illustrée, janvier 1916: 31). Une école est créée au dépôt des convalescents de Menton et un instituteur du cadre africain (enseignant exerçant en AOF), choisi parmi les auxiliaires, est désigné. Le but de cet enseignement est notamment de permettre aux tirailleurs de devenir des gradés, et d'être, de retour en Afrique, des agents utiles pour la France, tout en améliorant leur condition.

Dans un de ces camps d'hivernage, Lucie Cousturier obtient l'autorisation d'ouvrir une école où elle s'efforce d'enseigner le français standard aux tirailleurs. Elle écrit à propos $\mathrm{du}$ « jargon militaire » qu'ils emploient, dans une intéressante description critique de la méthode employée dans l'armée coloniale (d'autant plus intéressante qu'elle est une « voix discordante » précoce), qu'il

est issu de deux sources : celle d'abord des recrues bambaras qui ont indiqué, par leurs balbutiements en présence de notre langue, leurs préférences de formes et de 
mots; deuxièmement, celles des instructeurs blancs, qui ont adopté ces balbutiements et leurs conséquences pour principe de l'espéranto militaire. Supposons qu'ayant à enseigner notre langue à un Anglais, nous prenions soigneusement note des déformations que ses premières tentatives font subir à la syntaxe et à la prononciation française, et que nous nous basions sur elles pour lui présenter désormais un français réduit à ses comptabilités anglaises. Des résultats décevants de cette méthode il ne faudrait pas nécessairement conclure à une infirmité mentale des Anglais à l'endroit des langues [...]. (Cousturier 2001 [1920]: 82)

55 Les tirailleurs prennent conscience par le contact avec la population civile qu'ils parlent une variété qui n'est pas le «français de France » et qui en est même une variété ridicule; ils cherchent à s'échapper, lorsqu'ils en prennent conscience, de ce que Lucie Cousturier nomme une "prison verbale». Elle note ainsi à propos des tirailleurs qu'elle a rencontrés sur la Côte d'Azur, qu'ils « ont appris, par les rires, que leur langage les ridiculise : “c'est français seulement pour les tirailleurs"reconnaissentils tristement. Un de mes élèves, plus malveillant, assure que "c'est des mots trouvés par les Européens pour se foutre des Sénégalais ». (ibid. : 84)

Dix ans après la Première Guerre mondiale paraît le Règlement provisoire du 7 juillet 1926 pour l'enseignement $d u$ français aux militaires indigènes (1927), qui est en fait un manuel d'enseignement $d u$ français, selon la méthode directe ${ }^{16}$. Il y est notamment stipulé qu' « Il est formellement interdit de parler sabir (ou petit-nègre) », qualifiant cet usage $\mathrm{d}$ '« errements anciens » et pointant le fait que le petit-nègre ne remplit que mal ses fonctions, dans la mesure où il n'est guère plus simple que certaines formes simples de français standard (ce même manuel soulignant qu'il est moins difficile de dire : «balaie la chambre " plutôt que "toi y en abalayer la chambre »). C'est donc finalement un enseignement $d u$ français et en français qui va être mis en place au sein de l'armée coloniale.

\section{Ouverture: des langues spécifiques ou le français pour tous ?}

57 L'approche linguistique des autorités militaires vis-à-vis des soldats issus des colonies et notamment des colonies d'Afrique sub-saharienne (mais les problématiques seraient similaires concernant les soldats nord-africains ou asiatiques) semble être sous-tendue par deux types d'approche anthropologique : une approche culturaliste, qui privilégie l'usage des langues indigènes, la pratique de la traduction, ainsi qu'un Français « adapté », s'opposant à une approche universaliste, qui préconise le français standard au sein de l'armée. La fabrication du «français-tirailleur » semble être une réponse culturaliste à la question de l'utilisation du français par des «indigènes ». Cette approche culturaliste s'illustre par ailleurs dans d'autres entreprises, notamment dans la manière dont sont organisés certains camps d'hivernage. On peut citer la mise en place de lieux de culte spécifiques : par exemple, la reproduction, en béton armé rose, de la mosquée de Djenné, au Mali, dans un des camps de Fréjus, et la construction d'un temple bouddhique, dit pagode du camp Gallieni, érigé, également à Fréjus, par des tirailleurs indochinois. L'installation de certains centres d'hébergement des blessés, et notamment celui installé dans l'hôtel Carlton à Menton, dirigé par le docteur Maclaud, " parlant les dialectes de l'Afrique Occidentale Française », et conçu comme un " centre de resénégalisation » des tirailleurs, où il s'agit pour eux de retrouver leur mode de vie et leurs activités traditionnelles (ils y jouent à l'awalé, voir illustration 6, y organisent des «palabres» et des soirées de musique et de danse autour de «tam tam»). Le 
docteur Maclaud prend par ailleurs des initiatives d'ordre linguistique. Sur le mur d'un couloir, des écriteaux commandent «Makow!» (silence) en bambara. Et sur une lessiveuse transformée en «alcazaras ", une étiquette «trilingue » français/bambara/ petit-nègre indique : Boisson hygiénique - Cani - y a bon (décrit par Alphonse Séché : 245).

Mais, finalement, il semblerait que ce soit l'approche universaliste qui ait prévalu au sein de la hiérarchie militaire, s'illustrant linguistiquement par le choix d'utiliser et d'enseigner le français standard, contre l'usage des diverses langues indigènes et contre l'usage du petit-nègre. Cependant, cela ne veut pas dire que cette approche culturaliste n'ait pas marqué pour longtemps les pratiques au sein des troupes. Des témoignages tardifs rendent compte de la durabilité de l'usage du « sabir ».

Illustration 6 : tirailleurs sénégalais jouant à l'awalé (jeu traditionnel d'Afrique de l'Ouest), photographie extraite de La dépêche coloniale illustrée, février 1917 : « L'Afrique occidentale française et les troupes noires ".

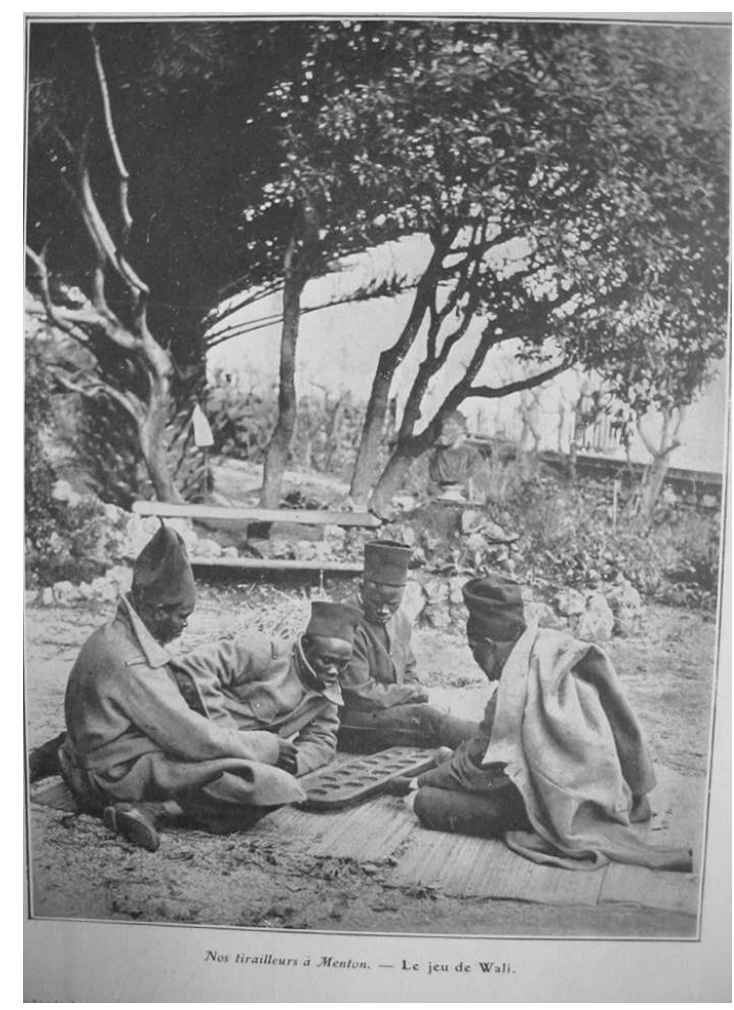

59 Ce qui se joue au sein de cette institution particulière qu'est l'armée, à travers ce que l'on peut décrire comme la mise en place d'une politique linguistique, rend donc compte de différentes façons d'envisager l'altérité au sein du système colonial, et n'est pas sans faire écho d'une part à la manière dont se constituent à la même époque l'anthropologie et l'ethnologie, et d'autre part à la spécificité de la politique coloniale française en Afrique Noire, laissant apparaître un penchant à l'assimilation républicaine, en tension d'autres pratiques, indigénisme et administration indirecte ${ }^{17}$. 


\section{BIBLIOGRAPHIE}

AMSELLE, Jean-Louis, SIBEUD, Emmanuelle. 1998. Maurice Delafosse, Entre orientalisme et ethnographie, l'itinéraire d'un africaniste (1870-1926). Paris : Maisonneuve et Larose.

ANONYME. 1916. Le français tel que le parlent nos tirailleurs sénégalais. Paris : Imprimerie Militaire Universelle L. Fournier.

AUROUX, Sylvain dir. 2000. Histoire des idées linguistiques. Liège : Mardaga.

BINGER, Louis-Gustave. 1886. Essai sur la langue bambara. Paris : Maisonneuve frères et C. Leclerc. BONNET, Gabriel 1941. Samba, héros de l'Empire. Paris : Sequana.

Bulletin du Comité d'études historiques et scientifiques de l'Afrique occidentale française, oct.déc. 1922, Paris : Larose.

CHAUDENSON, Robert. 2003. La créolisation : théorie, applications, implications. Paris :

L'Harmattan.

COUSTURIER, Lucie. 1920. Des inconnus chez moi. Paris : La Suène ; réédition. Paris : L'Harmattan, 2001.

DARD, Jean. 1825. Dictionnaire wolof et bambara. Paris : Imprimerie nationale.

DELAFOSSE, Maurice. 1901. Essai de manuel de langue mandé ou mandingue. Paris : Leroux.

DELAFOSSE, M. 1904. Vocabulaire comparatif de plus de soixante langues ou dialectes parlés à la Côte d'Ivoire. Paris : Leroux.

DeLAfosSE, M. 1914. Enquête générale des langues d'Afrique. Paris : Masson et C ${ }^{\text {ie }}$.

FERRAGE, le Père P.-M. 1918. Petit manuel français-bambara à l'usage des troupes noires. Paris : Imprimerie-Librairie militaire L. Fournier.

HOUIS, Maurice. 1984. « Une variété idéologique du français : le 'langage tirailleur'. Afrique et langage $21,1^{\mathrm{er}}$ semestre : 5-17.

LARCHAIN, Michel. 1923. «Enseignons le français aux indigènes. Pourquoi ne pas profiter, pour le faire, de leur séjour en France? ». La dépêche coloniale et maritime 7516, 13 janvier.

KAHN, Gisèle. 1990. « Un manuel pour l'enseignement du français aux militaires indigènes, 1927 ». Le français dans le monde. Recherches et Applications, août-sept. : 97-103.

MANGIN, Charles. 1910. La Force noire. Paris : Hachette.

MANNESSY, Gabriel. 1994. « Français tirailleur et français d'Afrique ». in Le français en Afrique Noire. Paris : L'Harmattan, 111-119.

MICHEL, Marc. 1992. «L'armée coloniale en Afrique occidentale française ». In Catherine COQUERYVIDROVITCH dir. L'Afrique occidentale au temps des Français. Colonisateurs et colonisés, c. 1860-1960. Paris : Éditions la Découverte.

MARC, M. 2003. Les Africains et la Grande Guerre. L'appel à l'Afrique (1914-1918). Paris : Karthala. Règlement provisoire du 7 juillet 1926 pour l'enseignement du français aux militaires indigènes. 1927 Paris, Limoges, Nancy : Charles-Lavauzelle et $C^{\mathrm{ie}}$, Éditeurs militaires.

SÉCHÉ, Alphonse. 1919. Les Noirs. D’après des documents officiels. Paris : Payot. 
TRAVÉLÉ, Moussa. 1910. Petit manuel français-bambara. Paris : Librairie Paul Geuthner.

\section{NOTES}

1. Pour une synthèse complète, voir Michel 1992.

2. Dès 1833, la citoyenneté est accordée à certains ressortissants libres de la colonie. En 1916, la citoyenneté française est élargie aux habitants de quatre communes du Sénégal (Saint-Louis, Rufisque, Gorée et Dakar). Les citoyens français bénéficient des mêmes droits politiques que les Français et ont un parlementaire à l'Assemblée nationale française. Les ressortissants du reste de l'AOF sont sujets français.

3. Le premier dictionnaire de bambara date de 1825 (Dard 1825); la première description est celle de Binger (1886).

4. Dont il est dit qu'elle est composée de «cent quarante recrues (des Mossis), soixante vieux noirs, anciens tirailleurs plus ou moins aptes à faire campagne, enfin, cinquante hommes environ venus du Sénégal, de Mauritanie, de la Guinée. Pas d'homogénéité. Instruction militaire plus qu'insuffisante » (Séché 1919 : 81-82).

5. Dans un ouvrage, où il propose une classification des langues d'Afrique, Delafosse classe le " petit-nègre » dans les « langues afro-européennes » au même titre que le broken-english, le sabir, le créole portugais, le créole français (1914).

6. Pour une présentation de l'itinéraire de Delafosse, voir Amselle / Sibeud 1998.

7. Extrait de Samba, héros de l'Empire (Bonnet 1941: 41), roman écrit par un capitaine des marsouins, qui retrace la destinée d'un jeune Malinké, fils d'un tirailleur ayant fait la guerre de 14-18. Les difficultés de traduction et les différents malentendus qui en résultent, de même que les différents cuirs produits par méconnaissance du français sont un des ressorts comiques largement exploités par ce type de roman que je surnomme romans «y a bon ».

8. Texte reproduit intégralement sur le site suivant : www.1914-1918.be/service sante_senegalais.php.

9. «Qu'on apprenne le français aux recrues alsaciennes, personne n’y trouve assurément à redire. Mais pourquoi n'apprendrait-on pas également le français aux recrues indigènes?» (Larchain1923).

10. Service Historique de l'Armée de Terre (SHAT), $26 \mathrm{~N}$ 871, JMO du $61^{\mathrm{e}}$ BTS.

11. Notes du 11 juillet 1917 et du 20 juillet 1917, SHAT, AG, 6 N 97.

12. 6 novembre 1917, SHAT, 6N97: Fonds Clemenceau, études diverses sur le personnel de l'armée 1916-1919.

13. SHAT, 6N97 : Fonds Clemenceau, études diverses sur le personnel de l'armée 1916-1919.

14. Il n'est d'ailleurs pas inintéressant de souligner que c'est dans des structures coloniales laissant davantage de place aux ecclésiastiques qu'a été favorisé l'usage des langues locales jusque dans l'instruction primaire. On peut ainsi donner l'exemple du Congo belge.

15. Déjà, dans un article paru le 22 septembre 1916 dans Les Annales coloniales, et rendant compte de la parution de l'ouvrage Le français tel que le parlent nos tirailleurs sénégalais, il est dit qu'il s'agit du sénégalais tel que le parlent les Français (cité par Michel 2003 : 116).

16. Pour une description de ce manuel, voir Kahn 1990 : 97-103.

17. À ce propos, lire par exemple la présentation que fait J.-L. Amselle de Faidherbe, conquérant, administrateur et ethnologue, inventeur de l'idée d'Afrique Noire, in Vers un multiculturalisme français. L'empire de la coutume 1996. Paris : Aubier, 117-150. 


\section{RÉSUMÉS}

Cet article se propose d'analyser la politique linguistique mise en place au sein de l'armée coloniale française, et plus précisément au sein des bataillons de tirailleurs dits "tirailleurs sénégalais » autour de la Première Guerre mondiale. La diversité des origines des tirailleurs (minoritairement sénégalais) a pour conséquence une grande diversité linguistique au sein des bataillons. D'autre part, les officiers, une partie des sous-officiers et les titulaires de spécialités techniques sont français. La question de la communication est donc à la fois un problème horizontal et vertical : elle concerne à la fois la communication quotidienne entre tirailleurs et la question, plus directement cruciale, de la compréhension des ordres. Les différents documents analysés ici rendent compte de deux types de pratique permettant l'intercommunication : d'une part l'usage de parlers véhiculaires, d'autre part l'usage de la traduction. Par ailleurs, ils révèlent, concernant le choix d'un véhiculaire, d'une hésitation et d'une pluralité de pratiques, une langue indigène pouvant être promue au rang de véhiculaire au sein de l'armée (c'est ce que nous verrons à propos du bambara), ou le français (dans une version éventuellement simplifiée) pouvant remplir ce rôle. Nous rendons compte ici à la fois d'articulations et de tensions entre ces diverses pratiques qui se révèlent à la lumière de l'engagement dans la Grande Guerre, et nous tâchons d'expliciter les conceptions anthropologiques qui les sous-tendent, dans la mesure où l'on peut considérer les pratiques militaires comme largement influencées par la manière dont le savoir ethnographique est en train de se constituer, au début du XXe siècle, en et sur l'Afrique de l'Ouest.

This article deals with the language policy of the French colonial army, more specifically as enforced within the battalions of tirailleurs known as tirailleurs sénégalais, around the First World War. The vast range of ethnic origins found among the tirailleurs - only a minority being Senegalese - creates an important linguistic diversity. On the other hand, the officers, a number of the NCO's and the technicians and engineers are French. Communicating is, as a result, both a horizontal and a vertical issue, involving the daily intercourse between the tirailleurs and, more importantly, the understanding of orders. The various documents which we analyse show two types of strategies allowing intercommunication : using vehicular languages and translating. Regarding the choice of a vehicular language, our documents show a variety of situations, with a vernacular sometimes selected, as we shall see with Bambara, or French, in some cases simplified, being chosen. We discuss connections and tensions between the various methods as monitored through the involvement in the First World War, and we try to show the anthropological biases which determine their choice, given the fact that one can consider army policies as greatly influenced by the path followed by ethnography, an emerging science at the start of the $20^{\text {th }}$ century, in and about Western Africa.

\section{INDEX}

Keywords : anthropology, Bambara, French colonial army, intercommunication, language policy, tirailleurs sénagalais

Mots-clés : anthropologie, armée coloniale française, Bambara, communication, Politique linguistique, tirailleurs sénégalais 
AUTEUR

CÉCILE VAN DEN AVENNE

ENS Lettres et sciences humaines / ICAR / Lyon 